\title{
Propuesta de normalización para la descripción petrográfica de materiales pétreos
}

\author{
Jesús Espinosa Gaitán \\ Departamento de Análisis. \\ Instituto Andaluz del \\ Patrimonio Histórico.
}

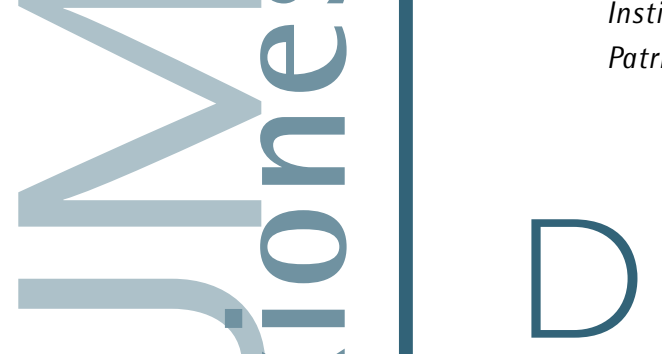

entro del marco del Programa de Normalización de Estudios Previos y Control de Calidad en las Intervenciones, que se está llevando a cabo en el I.A.P.H, se incluye la caracterización de los materiales empleados en las obras artísticas. Como es obvio, en este Proyecto se contemplan los materiales pétreos, de tan prolífera utilización en Bienes Inmuebles de nuestro Patriminio Artístico.

Al abordar un estudio de caracterización de rocas ornamentales, uno de los aspectos más significativos, para conocer su naturaleza y obtener una primera aproximación de su durabilidad, lo constituye la descripción petrográfica de dichos materiales. Esta descripción consiste básicamente en el estudio de la constitución y organización de los elementos que componen el material pétreo, incluyendo tanto elementos sólidos como espacios vacíos; siendo de especial interés en el campo de la Conservación las observaciones de las uniones intergranulares, presencia y naturaleza de matriz y/o cementos, posibles anisotropías texturales o granulométricas, así como el estudio de los espacios vacíos tanto en volumen como configuración.

Hasta la actualidad han sido escasos los intentos de realizar de forma normalizada los estudios petrográficos de materiales pétreos de uso ornamental. Cada especialista ha seguido sus propios criterios sin regirse a una descripción "ordenada", referida a unos mismos criterios descriptivos. Esto ha ocasionado que en el ámbito de los Bienes Culturales sea común encontrar descripciones notoriamente distintas sobre un mismo litotipo, tanto en los criterios como en las terminologías empleadas, suscitando la confusión entre descripciones realizadas por distintos autores.

En base a esto surge la necesidad de la elaboración de un guión descriptivo normalizado, con el que se pretende recopilar de forma esquemática, sin dejar de ser exhaustiva, la mayor información posible sobre las rocas de forma rápida, y a su vez de fácil comparación con otros litotipos.

La ficha que aquí se propone se refiere exclusivamente a rocas de origen sedimentario, que han sido las de mayor utilización en la construcción, al menos en Andalucía. Esta ficha, abierta a modificaciones y recomendaciones, se ha elaborado recopilandose criterios descriptivos de rocas detríticas y no detríticas, salvándose la posibilidad de dejar abandonados ciertos aspectos de interés en la descripción de rocas texturalmente mixtas entre ambos grupos, como es el caso de ciertas rocas carbonatadas.

Para su cumplimentación se ha revisado la bibliografía clásica especializada, así como otras propuestas de normalización recientes (NORMAL- 10/82; Vergerio P.P, 1995). La ficha se ha estructurado en cuatro bloques, que a su vez se dividen en diversos apartados. Estos apartados se proponen como modelo "ideal" de cumplementación; en muchos casos esto no será posible, bien porqué se desconozcan determinados aspectos, o bien porqué aquéllos no involucren a la roca en estudio.

A continuación se recoge de forma esquematizada la ficha con los distintos bloques y apartados, y posteriormente se exponen los criterios para rellenarla, así como la terminología propuesta en las distintas descripciones. 
FICHA DE DESCRIPCIÓN PETROGRÁFICA DE ROCAS SEDIMENTARIAS

\begin{tabular}{|l|}
\hline \multicolumn{1}{c|}{ ASPECTOS GENERALES } \\
\hline LITOTIPO \\
\hline NOMBRE Y SITUACIÓN DE LA CANTERA \\
\hline FORMACIÓN GEOLÓGICA \\
\hline AMBIENTE DEPOSICIONAL \\
\hline EDAD \\
\hline EJEMPLOS EN EDIFICIOS \\
\hline MORFOLOGÍAS DE ALTERACIÓN \\
\hline NOMBRE COMERCIAL \\
\hline
\end{tabular}

DESCRIPCIÓN MACRÓSCOPICA

COLOR

ESTRUCTURA

TAMAÑO MEDIO DE GRANO

TEXTURA GLOBAL

FRACTURAS/ FISURAS

OBSERVACIONES

\section{DESCRIPCIÓN TEXTURAL-MICROSCÓPICA}

MINERALOGÍA (\%)

ALOQUÍMICOS (\%)

TERRÍGENOS (\%)

ORTOQUÍMICOS (\%)

ASPECTOS TEXTURALES

ESPACIOS VACÍOS POROS

MICROFISURAS

CLASIFICACIÓN

FOLK

DUNHAM 
Descripción de los criterios y terminologías más comunes en su cumplimentación:

\section{ASPECTOS GENERALES}

\author{
I. LITOTIPO: \\ - Calcáreo \\ - Calcáreo-dolomítico \\ - Dolomítico-calcáreo \\ - Dolomítico \\ - Calcarenítico \\ - Detrítico: \\ - Rudita \\ - Arenita \\ - Lutita \\ - Mixto \\ - Otros
}

2. NOMBRE Y SITUACIÓN DE LA CANTERA:

Nombre con el que se conoce la cantera y situación geográfica de la misma.

\section{SITUACIÓN GEOLÓGICA:}

Ubicación y Formación Geológica a la que pertenece.

\section{AMBIENTE DEPOSICIONAL:}

Medio sedimentario en el que se formó la roca.

\section{EDAD:}

Edad geológica

\section{EJEMPLOS EN EDIFICIOS:}

Utilización en Monumentos Históricos.

\section{MORFOLOGÍAS DE ALTERACIÓN:}

Principales formas de alteración en cantera y en edificio

\section{NOMBRE COMERCIAL:}

Nombre con el que se comercializa dicha roca, si la cantera aún está en explotación, o nombre con el que comúnmente es conocida.

\section{DESCRIPCIÓN MACROSCÓPICA}

\section{COLOR:}

Estimación visual del color de la roca o empleo de la "Rock Colour Chart".

\section{ESTRUCTURA:}

- Homogénea

- Heterogénea

- Laminada

- Plano paralela

- Cruzada

- Ondulada

- Gradada

- Imbricada

- Isoorientada

- Bioturbada

- Estromatolítica

- Estilolítica

- Fenestral
- Nodulosa

- Micrítica

- Óxidos/hidróxidos/sulfuros

- Otros

- Con Microfracturas (Brechoide)

- Irregulares

- Orientadas

- Otras

3. TEXTURA GLOBAL:

- Clástica.

- Secuencial (Cristalina).

- Globular

- Grumosa

- Oolítica

- Organógena.

- Bioclástica.

- Mixta

- Otras

\section{TAMAÑO MEDIO DE GRANO:}

a. Tamaño absoluto.

- Muy grueso: $\varnothing>30 \mathrm{~mm}$

- Grueso: $\quad \varnothing: 5-30 \mathrm{~mm}$

- Medio: $\quad \varnothing: 1-5 \mathrm{~mm}$

- Fino: $\quad \varnothing<1 \mathrm{~mm}$

- Muy Fino: $\quad \varnothing<0,1 \mathrm{~mm}$

b. Tamaños relativos

- Equigranular

- Inequigranular

\section{FRACTURAS/FISURAS:}

Presencia de macrofisuras $y / 0$ fracturas.

- Anchura de apertura (valor medio).

- Longitud (valor medio).

- Selladas (naturaleza del cemento) y/o vacías

- Tipo:

- Intergranular

- Intercristalina

- Intragranular

- Intracristalina

- Transgranular

- Transcristalina

\section{OBSERVACIONES:}

Otros aspectos que se deban resaltar no incluidos anteriormente.

\section{DESCRIPCIÓN TEXTURAL-MICROSCÓPICA}

\section{MINERALOGÍA (\%) :}

Componentes minerales mayoritarios y minoritarios. Indicándose técnicas y métodos de identificación y cuantificación.

\section{ALOQUÍMICOS:}

(\% respecto total de los componentes texturales) Se debe indicar la naturaleza y porcentaje de cada uno de ellos.

- Bioclastos:

- Algas 
- Braquiópodos

- Briozoos

- Corales

- Equinodermos

- Foraminíferos

- Ostrácodos.

- Radiolarios

- Gasterópodos

- Artrópodos

- Otros

- Ovoides:

- Oolitos

- Ovoides Superficiales.

- Peloides:

- Pellets

- Gránulos amorfos

- Oncoides u Oncolitos

- Pisolitos

- Intraclastos

- Otros

\section{TERRÍGENOS:}

(\% respecto total de los componentes texturales)

- Componentes Terrígenos no Litoclásticos.

- Cuarzo.

- Feldespatos

- Otros

- Litoclastos (Fragmentos de rocas)

\section{ORTOQUÍMICOS:}

Matriz y Cementos. (\% respecto total de los componentes texturales). Se debe indicar la naturaleza y porcentaje de cada uno de ellos.

- Micrita: (Matriz carbonatada): $\varnothing$ cristal $<4 \mu \mathrm{m}$

- Relación entre cemento y matriz.

- Distribución:

- Homogénea.

- Heterogénea:

- Nodular

- Laminar o bandeado

- Orlas de revestimiento

- Reemplazamientos Intragranulares

- Esparita: (Cemento carbonatado): ø cristal > $4 \mu \mathrm{m}$

- Granular

- Mosaico (drusa)

- Fibroso o acicular

- Radioaxial

- Sintaxial

- Pecilítico

- Menisco

- Otros

- Microesparita: ø: 4-20 $\mu \mathrm{m}$

- Matriz y/o Cementos no Carbonatados:

- Tamaño:

- Fangoso: $\varnothing$ grano $<62 \mu \mathrm{m}$

- Granular: $\varnothing$ grano $>62 \mu \mathrm{m}$

- Mixto.

- Composición

- Silicatados:

- Arcilloso

- Opalino

- Microcuarcítico

- Ferruginosos:

- Óxidos e Hidróxidos de Fe, Mn, etc,
- Sulfuroso

- Glauconita

- Fosfatos

- Otros

Observaciones: diferenciación de la matriz fina originaria y cemento secundario.

\section{ASPECTOS TEXTURALES}

\section{A. Granulometría:}

\section{Rocas clásticas o mixtas}

(si se trata de materiales calacáreos o dolomíticos se le añade la raiz Calc-/Calci- o Dolo-).

- Rudita: $\quad \varnothing$ grano $>2$ mm

- Media: $\varnothing: 4-16 \mathrm{~mm}$

- Fina: $\varnothing: 2-4 \mathrm{~mm}$

- Arenita: $\varnothing$ grano: $2 \mathrm{~mm}-63 \mu \mathrm{m}$

- Gruesa: ø: 0,5-2 mm

- Media: ø: 0,25-0,5 mm

- Fina: $\quad$ : 0, I25-0,25 mm

- Muy fina: ø: $63 \mu \mathrm{m}-0,125 \mathrm{~mm}$

- Lutita: $\quad \varnothing$ grano $<63 \mu \mathrm{m}$

Rocas Carbonatadas Cristalinas:

- Fanerítica o Macrocristalina: $\varnothing>63 \mu \mathrm{m}$

- Esparita Megacristalina: ø cristales $>2 \mathrm{~mm}$

- Esparita Mesocristalina: $\varnothing$ cristales: 2 mm-63 $\mu \mathrm{m}$

- Afanítica o Microcristalina: $\varnothing<63 \mu \mathrm{m}$

- Esparita finamente cristalina: $\varnothing$ cristales: 63-20 $\mu \mathrm{m}$

- Microesparita: cristales: $\varnothing$ 20-4 $\mu \mathrm{m}$

- Micrita o Criptocristalina: $\varnothing$ cristales $<4 \mu \mathrm{m}$

\section{B. Morfología de los clastos:}

Grado de Redondez:

- Muy redondeados

- Redondeados

- Subredondeados

- Subangulosos

- Angulosos

- Muy angulosos

Grado de Esfericidad:

- Alta esfericidad o Isométricos

- Baja esfericidad o Anisométricos - Aplastado.

- Elongado.

- Otros

Formas Cristalinas (rocas cristalinas):

- Idiomorfos

- Hipidiomorfos

- Xenomorfos

\section{Empaquetamiento de los clastos.}

- Flotantes

- Puntual

- Tangentes

- Completo

- Suturado

\section{Sorting}

(Grado de desviación en tamaños de granos).

- Muy buen Sorting

- Buen Sorting

- Sorting Moderado

- Mal Sorting 


\author{
E. Madurez de la roca \\ Madurez Mineralógica \\ - Madura \\ - Inmadura \\ Madurez Textural \\ - Inmadura \\ - Submadura \\ - Madura \\ - Supermadura
}

\section{ESPACIOS VACÍOS}

\section{A. Porosidad}

Estimación Cuantitativa (\% volumen).

- Baja Porosidad: $n<5 \%$

- Porosidad Intermedia: n: 5-20\%

- Alta Porosidad: $n>20 \%$

\section{Tipos morfológicos:}

- Intergranular

- Intercristalina

- Intragranular

- Intracristalina

- Móldica

- Fenestral

- Shelter

- Framework

- Canalizada

- Vacuolar
- Brecha

- Otros

Relleno de poros

\section{B. Microfisuras:}

- Anchura (valor medio)

- Longitud (valor medio)

- Tipos morfológicos:

- Intergranular

- Intercristalina

- Intragranular

- Intracristalina

- Transgranular

- Transcristalina

- Orientación y Distribución:

- Isótropa

- Anisótropa (Indicar orientación)

- Relleno de microfisuras

\section{CLASIFICACIÓN}

\section{FOLK:}

- Clasificación de Rocas Carbonatadas (1959, 1962)

- Clasificación de Areniscas (1974)

\section{DUNHAM:}

- Clasificación de Rocas Carbonatadas (1962)

Bibliografía

ADAMS A.E., MACKENZIE W.S. \& GUILFORD. C (1984): Atlas of sedimentary rocks under the microscopy. Longman Scientific \& Technical.

CHOQUETTE, P.W and PRAY, L.C (1970): Geologic nomenclature and classification of porosity in sedimentary carbonates. Bull. Am. Ass. Petrol.Geol., 54, pp: 207-250.

DUNHAM, R.J ( 1962): Classification of carbonate rocks according to deposicional texture. In W. E:. Ham (Ed), Classification of carbonate Rocks. Mem. Am. Ass. Petrol. Geol., I, pp: 108-I2I.

FOLK, R.L (195I): Stages of textural maturity in sedimentary rocks. J. Sedim. Petrol., 21, pp: 127-130.

FOLK, R.L (1959): Practical petrographic classification of limestone. Bull.Am. Ass. Petrol,. Geol., 43, pp: I-38.

FOLK, R.L., (1962): Spectral subdivision of limestone types. In W. E:. Ham (Ed), Classification of carbonate Rocks. Mem. Am. Ass. Petrol. Geol., I, pp: 62-84.

FOLK, R.L., (1974): The natural history of cristaline calcium carbonate: effect of magnesium content and salinity. J Sedim. Petrol., 44, pp: 40-53.
FOLK, R.L., (1974): Petrology of sedimentary rocks. Hempphills, Austin. Texas.

LEIGHTON, M.W and PENDEXTER, C (1962): Carbonate rocks types. In W. E:. Ham (Ed), Classification of carbonate Rocks. Mem. Am. Ass. Petrol. Geol., I, pp: 33-6I

PETTIJOHN, F. J. (1975): Sedimentary rocks. Harper and Row, New York 3rd Edition.

Raccomendazione NORMAL (1982): Descrizione petrografica dei materiali lapidei Naturali. CNR-ICR. NORMAL - 10/82.

REIJERS, T.J.A. and HSÜ, K.J., ( 1986 ): Manual of carbonate sedimentology: A lexicographical approach. Academic Press, London, 302 pp.

VERGERIO, P. P e MEGGIOLARO, V., (1995): Proposta di schema standard per la descrizione e classificacione di litotipi carbonatici mediante analisi in microscopia ottica a luce trasmessa. Plinius, 14., pp: 305-306.

VERGERIO, P. P (1996): Descrizione petrografica dei materiali lapidei calcarei. Restauro \& Conservacione., 8, pp: 5-10. 
El patrimonio etnológico ha permanecido durante largo tiempo en el olvido, sometido a la indiferencia y la minusvaloración de quienes le concedieron un papel secundario en las acciones sobre el patrimonio. Ahora es el momento de dedicarle nuestra atención y considerar las reflexiones que los antropólogos hacen. " $\mathrm{Pa}$ trimonio vivo", "identidad”, “rehabitar", "reutilizar"....son palabras que nos sugieren aportaciones de plena vigencia. Las consideraciones recogidas en este número no quieren ser un homenaje aislado al patrimonio etnológico, sino una invitación a su continua presencia. 